UDC: 929 Baruh K. 929 Vidaković-Petrov K. 821.134.2:821.163.41(=411.16)

DOI: $\underline{\text { https://doi.org/10.18485/beoiber.2019.3.1.8 }}$

\author{
Miguel Rodríguez Andreu' \\ Doctorando en la Universidad de Valencia \\ España
}

\title{
KRINKA VIDAKOVIĆ-PETROV Y KALMI BARUH: UNA PERSPECTIVA BIOGRÁFICA INTERSUBJETIVA
}

Resumen

Krinka Vidaković-Petrov ha dedicado gran parte de su extensa carrera académica a estudiar la trayectoria de Kalmi Baruh. De hecho, la investigadora le considera "padre" de los estudios hispanistas en Yugoslavia. El presente artículo pretende analizar su vínculo personal con el intelectual bosnio-sefardí a través del método biográfico, recurriendo para ello a la asistencia a una de sus presentaciones académicas, a tres encuentros que se produjeron en Belgrado y a un documento personal redactado por ella misma. El objetivo del artículo, pese a las limitaciones de este modelo de investigación, es destacar la relevancia del trabajo realizado por Vidaković-Petrov y analizar los fundamentos cognitivos que justifican su interés por Kalmi Baruh en unas condiciones desventajosas, pero que evidencian en su persona una fuerte determinación política para dar voz al mundo sefardí después del Holocausto.

Palabras clave: Krinka Vidaković-Petrov, Kalmi Baruh, biografía, hispanismo, sefardí, Yugoslavia.

\section{KRINKA VIDAKOVIĆ-PETROV AND KALMI BARUH: AN INTERSUBJECTIVE BIOGRAPHICAL PERSPECTIVE}

\section{Summary}

Krinka Vidaković-Petrov has devoted much of her extensive academic career to studying the Hispanist Kalmi Baruh. In fact, the researcher considers him the "father" of the Spanish studies in Yugoslavia. The present article intends to analyze her personal connection with the Bosnian-Sephardic intellectual through the biographical method, including the attendance to one of her academic presentations, three meetings that took place in Belgrade and a personal document written by her. The aim of the article, despite the limitations of this research model, is to highlight the relevance of the work carried out by Vidaković-Petrov and to analyze the cognitive fundamentals that justify her interest in Kalmi Baruh in personally and professionally disadvantageous conditions, but which show a strong political determination to give voice to the Sephardic world after the Holocaust.

\footnotetext{
1 miguel roan@yahoo.es
} 
Key words: Krinka Vidaković-Petrov, Kalmi Baruh, biography, Hispanism, Sephardic, Yugoslavia.

\section{Introducción}

Mi interés por la profesora Krinka Vidaković-Petrov se sustancia a partir de sus investigaciones sobre el hispanista Kalmi Baruh y de mi campo de investigación, que se sitúa en el estudio de las cuestiones socio-políticas de la región balcánica, entre las que se encuentra la cultura e historia sefardí en el sudeste europeo. Teniendo mi residencia en Belgrado, el primer encuentro con ella se produjo en un congreso de hispanismo en la Universidad de Kragujevac el 29 de noviembre de 2014. Ante el público expuso sus investigaciones. Aquella impresión fue memorable: la profesora logró captar mi atención con una ponencia sobre la biografía del hispanista sefardí y una aproximación al inicio de los estudios hispanos modernos en Yugoslavia. A partir de ahí, se produjeron tres encuentros más. El 10 de julio de 2015 en una cafetería de Belgrado; el 17 de diciembre del 2015 durante la presentación del número 6 de la revista Balkania², de la cual soy editor desde el año 2010, y en la que participó la propia Vidaković-Petrov como autora de un artículo sobre las adivinanzas judeo-españolas en Macedonia3; y el 13 julio de 2016 en otra cafetería de Belgrado. Este número de la revista Balkania estaba dedicado a la cultura sefardí en los Balcanes occidentales y fue coordinado por la profesora Jelena Filipović, jefa del Departamento de Estudios Ibéricos en la Facultad de Filología de la Universidad de Belgrado, que me introdujo de una manera más profunda en esta temática, y me posibilitó entrar en contacto con diferentes autores especializados en la materia, entre los que se incluye la propia Krinka Vidaković-Petrov.

Para la elaboración de la presente investigación se ha recurrido a estos encuentros. Del mismo modo, pude compartir varios correos con ella, que sirvieron también para hacer un mapa mental sobre la trayectoria, intereses y motivaciones de la profesora Vidaković-Petrov. En concreto, en uno de ellos, iba adjunto un largo documento word, titulado "Krinka Vidaković-Petrov. Una historia que empieza como aventura y termina en nada nuevo" (Vidaković-Petrov 2018), en el que relata algunos de los pasajes más importantes de su carrera profesional que la unen con la figura de Kalmi Baruh. Este correo electrónico estaba destinado a aportar información para la publicación del libro Maratón balcánico (Caballo de Troya 2018), del que soy autor, pero por cuestiones de edición, la naturaleza del documento y el valor del mismo he preferido dedicarle un artículo más extenso y minucioso. El objetivo es poner en valor el trabajo académico de la investigadora y contribuir con ello a un conocimiento más complejo y certero sobre el estudio de la cultura sefardí en la antigua Yugoslavia.

2 En el Instituto Cervantes de la capital serbia.

${ }^{3}$ V. Vidaković-Petrov 2015.

BEOIBERÍSTICA Vol. III / Número 1 (2019) | 121-135 


\section{Cuatro limitaciones del estudio biográfico}

1) Este tipo de análisis está basado en el "método biográfico" (Denzin 1989; Pujadas 2002; Sanz Hernández 2005). Por sus limitaciones, hay muchas variables que no pueden ser evaluadas conforme a criterios estrictamente científicos, por no ir acompañadas de los suficientes fundamentos documentales ni tener garantizados todos los elementos exigibles de objetividad plena. He tenido un acceso reducido a la protagonista, aunque considero la información recopilada como una base suficientemente sólida para construir una unidad explicativa, que pueda interesar a futuros investigadores sobre la personalidad de Vidaković-Petrov y los estudios sefardíes.

2) Este trabajo se entroncaría con los estudios antropológicos, sociológicos y psicológicos, en tanto que se aporta un relato retrospectivo aportado por la profesora Krinka Vidaković Petrov, que constituye la narración de la propia vida contada por su protagonista desde un punto de vista idiográfico. Sumado a ello, se incluyen mis intervenciones intersubjetivas. Mis investigaciones y observaciones sirven para dotar de contexto situacional a su narración personal. De acuerdo con Allport (1942), el documento personal es un escrito de cualquier naturaleza que ofrece información sobre la naturaleza y los contenidos del pensamiento de su autor. No obstante, siguiendo los apuntes de Pujadas (2002), hay que destacar que este documento (Vidaković-Petrov 2018) estaba condicionado por mi solicitud de información, lo que, en este caso, canaliza la información aportada hacia la comprensión del vínculo de la autora con Kalmi Baruh. Esto puede suponer que la propia autora haya renunciado a ciertos datos que eventualmente pudieran ser relevantes para la investigación desde un punto de vista holístico.

3) La narración personal en algunas fases puede ser interpretada como representacional porque se destacan contextos de la etapa yugoslava que incidieron sobre las decisiones tomadas por Vidaković-Petrov. Este artículo, sin embargo, no busca establecer patrones comunes a partir de una sola voz, ni que la individualidad radical de la investigadora pueda ser tomada como modelo de una época. Las circunstancias sociales vividas por la profesora pueden ser generales, pero las presiones pueden ser meramente personales o no compartidas (Fox 1981). Por este motivo, el análisis del proceso de acercamiento a la figura de Kalmi Baruh solo pretende recoger cómo Vidaković Petrov ejerció su actividad "frente a las constricciones del medio y cómo sus diferentes experiencias se [fueron] concatenando en respuesta a ellas, desenredando así los vectores generales y particulares que engloban" (Capriati 2017: 320). Esto requiere de instrumentos de análisis diferentes que estudien y contextualicen las motivaciones, las resoluciones personales y los resultados, pero que no ambicionan salirse del personaje, sino dotarle de coherencia en un espacio de interacción político-social más amplio y complejo. 
4) El recurso a un documento temático aporta un objeto de análisis limitado en otra dimensión. Es un testimonio relevante sobre las propias percepciones personales de Vidaković-Petrov, que arroja exhaustividad y objetividad a la investigación, expresando "el recuerdo de lo acontecido, filtrado, como es lógico, por las emociones vividas en el momento y por las autolecturas de los episodios pasados en función de las resignificaciones que vamos construyendo acerca de nosotros mismos en nuestros sucesivos períodos vitales" (Capriati 2017: 321). La potencialidad de esta perspectiva tiene una objeción complementaria desde el momento en que es tasada por mí mismo: la intersubjetividad determina una selección interesada de acontecimientos expresados en la autobiografía; pero el objetivo de este artículo no es entender el pasado, ni traerlo al presente para analizarlo, sino dejar constancia de los estímulos con los que Vidaković Petrov desempeñó su tarea y reconstruir una experiencia personal que se asume relevante desde un punto de vista estrictamente epistemológico.

Sin embargo, el valor del relato aportado por Vidaković-Petrov y la trayectoria profesional de la investigadora, compensan las desventajas de este tipo de análisis, una vez el testimonio personal no solo esclarece las propias motivaciones de un actor relevante de este campo de investigación, sino que ofrece una descripción del entorno en el que se produjo su actividad profesional y qué acontecimientos determinaron sus decisiones. De esta manera, este texto puede aportar una fuente más de información, de la mano de uno de sus protagonistas, acerca de la evolución de los estudios sefardíes en la ex Yugoslavia y en los Balcanes occidentales, reconociendo, además, el papel jugado y las aportaciones realizadas por otros investigadores. Además, este artículo ofrece una perspectiva interdisciplinar, que puede aportar un interesante material para futuras investigaciones que no tienen por qué estar necesariamente vinculadas con los estudios sefardies.

\section{Las constricciones académicas}

Krinka Vidaković-Petrov nació en Belgrado en 1949. Siendo hija de un diplomático yugoslavo (Boško Vidaković), vivió en Chicago, El Cairo y La Habana. Recién llegada desde la Habana, las inquietudes de Vidaković-Petrov iban desde la poesía afrocubana hasta su pasión por el poeta Pablo Neruda. Estaba claramente orientada al hispanismo, aunque sopesó también la idea de estudiar Arquitectura. Finalmente, quiso matricularse en la Facultad de Filología en la Universidad de Belgrado. Según ella misma recalca:

En esa época eso resultó imposible porque la lengua española se podía estudiar sólo 4 semestres y con nada de literatura... En la facultad me aconsejaron escoger otro departamento de lengua-literatura para poder pasar al departamento de español en cuanto lo establecieran. Así que estudié lengua inglesa y literaturas anglófonas (Vidaković-Petrov 2018). 
Pese a las buenas perspectivas académicas que podía tener, como hablante de español, de acuerdo con la versión de Vidaković-Petrov, no pudo realizar los estudios de máster en la Facultad de Filología porque no fue admitida por la jefa del hispanismo de la Facultad de Filología de Belgrado, la profesora Ljiljana Pavlović-Samurović. Así que, terminada su licenciatura en Lengua Inglesa y Literaturas Anglófonas, y sin que hubiera todavía cátedra de español, su mentor fue el profesor Vladeta Košutić, profesor del Departamento de Literatura Mundial y Teoría Literaria, "que en esa época estaba traduciendo las obras completas de F. García Lorca" (Vidaković-Petrov 2018). Al no tener acceso al Grupo de Lengua y Literatura Españolas del Departamento de Romanística, una vez terminados sus estudios de máster sobre poesía afrocubana, su relación académica con la Facultad se termina. En este punto, Vidaković-Petrov, ya había comenzado su carrera profesional en el Instituto de Literatura y Arte en Belgrado como pripravnik ${ }^{4}$, para dos años después conseguir un puesto en el Departamento de Teoría de la Literatura.

El relato de su historia pone énfasis en las dificultades institucionales, pero también en las carencias estrictamente académicas de su campo de investigación. Reconoce como "romántica y utópica" la idea de estudiar la poesía de Pablo Neruda, ya que en los centros de investigación de la época escaseaban las fuentes bibliográficas sobre hispanismo. Considera como clave el papel del profesor Vladeta Košutić, que le dijo: "si quería dedicarme a la literatura de lengua española, TENÍA ${ }^{5}$ que ir a España. Punto" (VidakovićPetrov 2018). De las palabras de Vidaković-Petrov se deduce la solidez de los cimientos precursores a partir de los cuales se labra la carrera de una investigadora. Se trata de un modus operandi típico de cualquier investigador, pero que en su caso se expresa con una particular intensidad: acudir al terreno para que la investigación se acorde con los criterios científicos, tener acceso a las fuentes y entrar en contacto con las referencias académicas en la materia de estudio.

Vidaković-Petrov viajó por primera vez a España en 1972. Señala que: “Mientras los demás yugoslavos iban a ver las atracciones turísticas, yo iba a Madrid a explorar cómo iba a hacer mi tesis doctoral sobre los sefardíes" (Vidaković-Petrov 2018). El tono de sus afirmaciones denota tanta soledad y aislamiento en su misión, como una fuerte determinación, lo que queda todavía más acentuado por un elemento contextual: la ausencia de vínculos académicos hispano-yugoslavos. Hay un hecho anecdótico, pero no por ello menos gráfico, que reafirma esta realidad: tuvo que enviar su pasaporte a la Embajada de España en Roma para poder realizar el viaje.

Para Vidaković-Petrov, el ámbito académico es un escenario que demanda de su iniciativa como emprendedora académica. Esto se constata durante su incursión en los estudios sefardíes a comienzos de los años 70. Dice: "Cuando fui a la Comunidad judía para preguntar por materiales, se extrañaban mucho: si ellos mismos no se interesaban

\footnotetext{
${ }^{4}$ Empleado sujeto a contrato temporal.

${ }^{5}$ Las mayúsculas, como en el resto de citas de Vidaković-Petrov, respetan el texto original.
} 
por ese tema, iqué hago yo preguntando por ello?" (Vidaković-Petrov, 2018), o durante su estancia en Sarajevo para realizar su investigación: "El bibliotecario me miró con asombro y consternación. Me comentó que NADIE había pedido esos periódicos desde la posguerra (cuando él se hizo empleado allí)" (Vidaković-Petrov 2018). Son fricciones que se repiten con regularidad en su narración. La singularidad de los estudios que estaba realizando es una desventaja pero también dan forma a una personalidad que se construye a partir de esa adversidad, donde no parece haber virajes ni giros existenciales que desvíen la dirección de su vocación investigadora.

Si se hace un balance de los primeros años de carrera académica se puede observar por un lado que Vidaković-Petrov siempre fue por delante de los progresos institucionales que la propia Facultad de Filología de Belgrado fue realizando hacia la consolidación de los estudios hispanistas. El primer curso de Lengua y Literatura española como licenciatura se inauguraba en la Facultad de Filología en 1971. Los primeros estudiantes se titularon en 1975 y el primer doctorado en letras españolas fue otorgado en 1984 (Soldatić 2010: 25). Ante la imposibilidad de poder hacer el doctorado en la Universidad de Belgrado, terminó defendiendo su tesis a través de la Universidad de Zagreb. La propia VidakovićPetrov declara: "Tardé diez años en escribir y defender mi tesis (en 1982)" (VidakovićPetrov 2018). Este contexto es importante, porque su proyecto profesional fue tomando cuerpo al margen de la institución académica de mayor autoridad en aquel momento en Yugoslavia, incluso iniciando su investigación sobre los estudios sefardíes sin un mentor académico: “Un profesor de Zagreb, al cual conocí en una conferencia organizada por la Academia en Sarajevo, me ofreció averiguar si podría registrar mi tema como tesis doctoral en Zagreb. $\mathrm{Y}$ así me puse en contacto con mi futuro mentor en Zagreb" (Vidaković-Petrov 2018).

Las desventajas que sufrió la investigadora encajan con el relato compartido y su personalidad donde resuelve los problemas que se le plantean gracias a la propia iniciativa, pero también al contacto, motu propio, con terceras personas a las que otorga un papel fundamental en el devenir de los acontecimientos. Su primera conexión con los estudios sefardíes surge a partir del encuentro con dos personas. Primero, Simha Kabiljo Šutić, una colaboradora del instituto donde trabajaba, que había perdido a muchos de sus familiares -sefardíes de Sarajevo- en los campos de concentración organizados por los ustaše croatas, y que hablaba algunas palabras en judeoespañol. Y, también, Žarko Vidović, jefe del departamento y amigo de su padre, serbio de Sarajevo, que había sobrevivido a varias deportaciones y campos de concentración durante la Segunda Guerra Mundial.

Del mismo modo, se desprende que su obstinación obtiene su premio una vez se inicia su vinculación con los estudios sefardíes en España, manifestándose tras el acceso a instituciones especializadas de las que había carecido en Yugoslavia en los primeros años de su carrera académica; esto es el Consejo Superior de Investigaciones Científicas y la Cátedra-Seminario Ramón Menéndez Pidal, con las que entró en contacto durante su segundo viaje a España. Durante esta estancia en Madrid, conoció al profesor Jacob 
Hassan y al profesor Diego Catalán que tuvo, según ella, un papel determinante en la obtención de una beca del gobierno español en 1974, cuando las relaciones entre España y Yugoslavia eran inexistentes. El círculo de relaciones se amplía a otros nombres de los que deja constancia: "Mi vínculo con unos fueron los sefardíes, con otros el romancero, con todos la curiosidad, el diálogo, la amistad" (Vidaković-Petrov 2018).

Dentro de su propia carrera en los estudios sefardíes, el trabajo de análisis de la figura de Kalmi Baruh tiene su inicio en un contexto muy específico, pero, igualmente, estimulado por el contacto con terceras personas. Ella lo narra así:

Gracias al café y el tabaco (dos leitmotifs de esa época, no sólo de los Balcanes) conocí a un miembro de la academia de $\mathrm{BiH}$, ilustre y viejo, muy conocido, que hablaba con voz baja, llevando en la cara una sonrisa triste. Yo no sabía al principio quién era ese señor amable, pero me di cuenta de que sabía mucho sobre los sefardíes de Sarajevo. Me invitó a escribir un artículo sobre el estado de la investigación de ese tema en Yugoslavia. Y aquí entra en mi historia Kalmi Baruh. Sobre lengua y literatura sefardí no había CASI nada en la época de la posguerra. Lo fundamental era lo que había hecho Baruh en la época anterior al Holocausto. De allí mis primeras publicaciones sobre el tema ${ }^{6}$ (Vidaković-Petrov 2018).

En este pasaje se está refiriendo al profesor Midhat Begić (1911-1983), historiador y especialista en Literatura que, entre otros desempeños profesionales, fue profesor en la Universidad de La Sorbona (1968-1969).

En definitiva, la trayectoria académica de Vidaković-Petrov está marcada por una suma de decisiones de naturaleza estrictamente individual, pero siempre entretejidas con un entramado de encuentros casuales o buscados con diferentes personas que impulsarán, pero también irán conformando el rumbo académico de la investigadora.

\section{Las motivaciones políticas}

Las motivaciones políticas son un elemento trascendental en el perfil vital de Krinka Vidaković-Petrov, y están enlazadas con la trayectoria profesional de la investigadora. Era una niña cuando fue testigo del cambio de gobierno en Cuba -la dictadura de Fulgencio Batista fue derrocada por la Revolución cubana-. Son aspectos que ella destaca como cruciales en su infancia. Ella, en su texto autobiográfico, hace referencia al "primer contacto con "Rusos españoles", la primera lectura del Puente sobre

${ }^{6}$ Estas son (,Kalmi Baruh o Sefardima“, Izraz, 1976, 5, 801-811; „Mogućnosti proučavanja jezičkoknjiževnog nasleđa španskih Jevreja na našem tlu“, Forum, 1976, 7-8, 302-312; „Jevrejsko-španski listovi u Bosni i Hercegovini“, Mostovi, 1977; „Sefardska književnost u Bosni i Hercegovini u svetlu dosadašnjih istraživanja“ Akademija nauka i umjetnosti BiH, Naučni skup Književnost BiH u svijetlu dosadašnjih istraživanja, Posebna izdanja XXXV, knj. 5, Sarajevo, 1978, 85-92). 
el Drina de Andrić, el Che en nuestra casa, Djilas en la portada de Lifes, (Vidaković-Petrov 2018). Son episodios que la conectan con un paisaje de naturaleza política, que encaja, además, en la idea yugoslava de la posguerra europea, donde la política nacional estaba asociada a la victoria partisana sobre el fascismo, pero donde también el propio sistema yugoslavo sufre impugnaciones contra su legitimidad política, que se manifiestan a través de su independencia ideológica y geopolítica frente a los sistemas de tipo soviético o la ideología liberal-capitalista durante la Guerra Fría.

La generación de Vidaković-Petrov fue la primera generación realmente yugoslava dentro del socialismo, nacida tras las Segunda Guerra Mundial. Es importante destacar que su vuelta a Belgrado, una vez terminados los estudios en el barrio habanero de Vedado, coincide con un periodo de expansión intelectual en Yugoslavia, marcado por la condición del país balcánico como líder del Movimiento de Países No Alineados. Eran los tiempos en los que Yugoslavia creaba nuevos puentes asiáticos, latinoamericanos y africanistas que hicieron de esa nueva generación universitaria una vanguardia cultural que superaba la división geopolítica entre dos bloques, para conectarse con ámbitos de conocimiento hasta entonces desconocidos o ajenos a la tradición investigadora yugoslava. Esta tendencia estaba afianzada por las propias libertades culturales que el propio sistema yugoslavo permitía en contraposición al escenario soviético.

De toda esta experiencia vital se presume su afinidad hacia la idea yugoslava y cómo su trayectoria encaja en ese esquema identitario aunque no fuera siempre una circunstancia buscada, repartiendo sus referencias geográficas para la investigación entre la comunidad judía en Sarajevo, el Instituto donde trabajaba en Belgrado y su condición de doctoranda en la Universidad de Zagreb. El caso de Vidaković-Petrov solo viene a confirmar la existencia de una primera generación de yugoslavos de la etapa socialista cuya condición les identificaba con otros colectivos, como la comunidad sefardí, por la que nuestra autora siente una fuerte atracción intelectual y personal gracias a su pasión por el hispanismo. Al respecto, en su trayectoria profesional Vidaković-Petrov también destaca por haber sido galardonada con el premio Miloš N. Đurić, a la mejor traducción poética por Izabrane Pesme Rafaela Albertija (Poemas seleccionados de Rafael Alberti), en 1984, autor exiliado durante la época franquista y que se acopla a los preceptos reivindicativos de la lucha antifascista yugoslava, pero también a la cosmología y vocación global que propugnaba el no alineamiento de Yugoslavia.

El descubrimiento del mundo sefardí, igualmente, se sustancia a partir de una conciencia subjetivada en el contexto político yugoslavo. Mis encuentros con VidakovićPetrov manifestaron la motivación política como un rasgo de su personalidad como investigadora, pero que intervenían sobre ella de una manera exógena, en clave reactiva: "A mi no me interesaba la política, ella se interesaba por mí" (Vidaković-Petrov 2018). Consiste en un contexto preexistente que no ignora, sino al que se agarra para confrontarlo a su manera y de acuerdo a sus propios ideales. De hecho, el acercamiento de Vidaković-Petrov a los estudios sefardíes se produce pocos años después de la rotura de relaciones de Tito con Israel tras la Guerra de los 6 días (1967). Las circunstancias 
políticas cuestionan el ánimo de Vidaković-Petrov, que en todo momento se expresa en ella a través del impacto que va teniendo la política sobre su carrera académica: su padre fue acusado oficialmente en 1973 de "propaganda enemiga", siendo ella una investigadora que proyectaba sus inquietudes personales hacia un país al que la política nacional catalogaba de "fascista" (España); se vio inmersa en la generación involucrada en las manifestaciones de junio del 68 , que sufrió durante los años 70 sucesivas purgas y restricciones de libertades públicas; la política exterior yugoslava estuvo orientada o fue manifiestamente pro-árabe mientras sus vínculos con el mundo judío comenzaban a estrecharse; incluso en su documento relata como durante la primera presentación de su libro en Sarajevo (1986) hubo un aviso de bomba que se puede asociar a una atmósfera de crispación que el conflicto israelí-palestino provocaba entre las diferentes sensibilidades políticas locales. Cada uno de estos episodios demanda de Vidaković-Petrov una respuesta individual que se articula a través de su compromiso con su trayectoria profesional.

La actitud de Vidaković-Petrov muestra su activismo intelectual contra las injusticias históricas, un compromiso con una época que no le tocó vivir pero cuyos ecos la empujan al posicionamiento político. Señala al respecto: “Para mí, el tema de los sefardíes era un diamante olvidado, despreciado e invisible bajo varias capas de lodo y basura histórica. Nadie lo veía, nadie le daba importancia" (Vidaković-Petrov 2018). De las conversaciones mantenidas con Vidaković-Petrov, como también del rumbo de sus investigaciones, entre las cuales destacan, por ejemplo, sus trabajos sobre los serbios en América, se presume su voluntad de conectar el drama judío y el serbio, como dos naciones que sufrieron persecución y campañas de aniquilación física durante el siglo XX. La reivindicación del reconocimiento y homenaje a los serbios víctimas de las políticas del Estado Independiente de Croacia y de la ocupación nazi durante la Segunda Guerra Mundial es un tema que adquirirá cada vez más importancia dentro de Yugoslavia, como una cuenta pendiente que las instituciones socialistas tenían con la sociedad serbia respecto al genocidio ustaše, sus responsables políticos y militares, y el consiguiente homenaje a las víctimas.

Esta motivación no esta carente de elementos de naturaleza personal en sentido estricto, más allá de las cuestiones de identificación empática que se manifiestan de forma voluntaria. Un factor que ella destaca como relevante, en una entrevista que le realizaron (Pravoslavlje 2018), fue la muerte de su bisabuelo, el sacerdote Veljko Tankosić, colgado de la horca por los austriacos en 1916, en Užice, hecho que ella se encarga de destacar como un suceso que no es para nada extraordinario en su contexto nacional: "cada familia serbia tiene una historia similar" (Pravoslavlje 2018: 29), pero que en su caso permite establecer un desarrollo lógico entre sus orígenes familiares, los estudios sefardíes y el mundo serbio. En la misma entrevista, se encarga de destacar que no existe ningún tipo de reconocimiento o memorial en su ciudad (Belgrado) donde se pueda presentar respetos a las víctimas de Jasenovac, "el mayor patíbulo de la nación serbia durante la Segunda Guerra Mundial” (Pravoslavlje 2018: 26). De hecho, en la entrevista 
mencionada, cuando le preguntan por la variedad de intereses que muestra su carrera profesional, hace referencia al cuento "La noche serbia" del escritor y periodista español Blasco Ibáñez, donde se hace mención a la Albanska Golgota (Pravoslavlje 2018: 26), cuando el Ejército serbio, durante la Primera Guerra Mundial, se retiró a través de las montañas albanesas en dirección a la isla griega de Corfú, pereciendo más de 200.000 personas en la travesía. Se puede concluir, por tanto, que la investigadora encuentra en su pasión por el hispanismo un mecanismo más para materializar sus motivaciones políticas, donde cohabitan su interés por el mundo sefardí y por el destino de la nación serbia a la que pertenece, como partes de un todo político y emocional. Un apunte de su biografía, por ejemplo, donde encajan todas estas piezas personales, ideológicas y profesionales fue su condición de Embajadora de Yugoslavia entre el año 2001 y 2006.

\section{Descubriendo a Kalmi Baruh}

Siguiendo la biografía de Kalmi Baruh se pueden apreciar algunos elementos en común con la investigadora, pese a las distancias espaciales, temporales y contextuales, pero que establecen una identificación personal que puede ayudar a entender el vínculo intelectual que Vidaković-Petrov constituyó con el hispanista sefardí. Baruh pertenece a una primera generación de bosnios que estudiaron en las escuelas austro-húngaras y que conectaron con el mundo centro-europeo, en un tiempo de aperturismo, pero también de limitaciones estructurales. Baruh terminó su tesis en 1923 en la Universidad de Viena con el título Der Lautstand des Judenspanischen in Bosnien, que trataba de la lengua sefardí de Bosnia. Sin embargo, su actividad profesionalidad, al igual que la de VidakovićPetrov, abrió una nueva vía académica, ambos en una fase de renacer del mundo académico después de la Primera Guerra Mundial y después de la Segunda Guerra Mundial respectivamente.

Baruh se erigió como la voz más destacada del hispanismo de aquella época, pese a no ser profesor universitario, cuando España y la Yugoslavia monárquica eran prácticamente dos desconocidos. A su vuelta a Sarajevo, donde trabajó como profesor del Liceo francés, Baruh desempeñó una incansable actividad intelectual, rodeándose de la élite yugoslava de aquel momento, y difundiendo la cultura sefardí y española en multitud de publicaciones, foros y conferencias. Formó parte de un exclusivo grupo sefardí con estudios en el extranjero: "los dotores". Al igual que Vidaković-Petrov, ambos acudieron a España para progresar en sus estudios en una época donde los puentes institucionales no existían.

La afinidad que siente Vidaković-Petrov se explica, de acuerdo con mis propias interpretaciones, no solo a partir de la relevancia académica del hispanista, sino también por el destino trágico de Baruh durante la Segunda Guerra Mundial. En 1941, durante la Segunda Guerra Mundial, los ustaše croatas fundaron el Estado Independiente de Croacia, gracias a la ofensiva nazi, y ocuparon Bosnia-Herzegovina y parte de Serbia. Le siguió una 
campaña de exterminio de la población judía, serbia y romaní. Baruh consiguió escapar a Montenegro, controlado en aquel entonces por el Ejército italiano, pero dos años después, tras la capitulación del país ítalo, Baruh sería detenido y enviado a Bergen Belsen, donde moriría cinco días después de la liberación del campo de concentración. Cuenta uno de los testigos, Isaac Levy, que, durante su estancia en el campo, Baruh estuvo impartiendo clases de español y recopilando materiales y entrevistando a los presos de origen sefardí con los que compartía barracones. Esto demuestra una fuerte resolución por el trabajo desempeñado, como demuestra también el caso de VidakovićPetrov, aunque las circunstancias no sean en ningún caso comparables. No resulta extraño que el trabajo de la investigadora, concienciada de la tragedia del holocausto nazi sobre los judíos, se oriente hacia el drama humano sufrido por Baruh, como judío, desde una perspectiva colectiva, pero también desde una perspectiva individual, tanto a nivel de la pérdida de un excelente hispanista, como de la perdida de una excelente persona. Ella misma se encarga de destacar este extremo (Vidaković-Petrov 2016) a través, por ejemplo, de Giménez Caballero: "él es el único elemento valioso de que disponemos en el próximo Oriente para una acción cultural eficiente" (apud Vidaković-Petrov 2016: 166) o del nobel serbio Ivo Andrić, quien destacó ante todo el humanismo, dedicación, amistad y modestía de Kalmi Baruh (apud Vidaković-Petrov 2016: 162). La investigadora dice así:

La contribución de Baruh a los estudios en el campo sefardí e hispánico, sus traducciones de literatura española, sus conferencias públicas y artículos periodísticos, los cursos de español que dictó en Sarajevo en 1935 gratis, la modestia con la cual rechazó la oferta de ser el primer profesor de la cátedra de estudios hispánicos en Belgrado, incluso sus actividades en el campo de concentración Bergen Belsen hablan de un hombre excepcional dedicado a una misión noble. Baruh nunca llegó a ser un profesor universitario, pero es, sin duda, el padre de los estudios hispánicos en Yugoslavia. (Vidaković-Petrov 2016).

Resulta oportuno referirnos a algunas de las percepciones expresadas por la propia profesora en publicaciones de su autoría, acentuando aquellas cuestiones que otros investigadores han aportado, como es el caso del artículo en serbio "Temas judíos en los trabajos de Vojislav Maksimović" (Vidaković-Petrov 2018) ${ }^{7}$. En este artículo Vidaković-Petrov pone énfasis en la contribución de Maksimović al conocimiento de la figura de Baruh, subrayando no solo las aportaciones académicas del hispanista, sino también "su participación relevante en la vida cultural de la comunidad bosnio-judía", especialmente en un momento en que la comunidad sefardí y askenazí desarrollaban su actividad en el espacio yugoslavo y eran necesarios líderes intelectuales. Y, al mismo tiempo, señala el hecho de que Baruh fuera capaz de abarcar todo tipo de temáticas vinculadas a España, su cultura

\footnotetext{
7 Jevrejske teme u radovima Vojislava Maksimovića.
} 
y su momento político contemporáneo, especialmente durante la Guerra Civil, sobre la que dejó plasmados sus pensamiento, pero también sobre los judíos en el marco del espacio europeo. De hecho, Vidaković-Petrov en este texto, pone en valor como Baruh era más que consciente del mal que acechaba a los judíos en Alemania con la llegada de Hitler al poder.

Se puede asumir que la investigadora sintió un enorme interés por la figura de Kalmi Baruh desde prismas muy diferentes, que abarcan desde su trayectoria académica, sus éxitos profesionales, su historia de vida, sus vínculos sociales o personales, hasta su muerte en unas circunstancias tan dramáticas para la comunidad judía como fue el Holocausto nazi, que resultó en la desaparición de más de un $75 \%$ de los judíos yugoslavos. Todas estas facetas han sido analizadas por VidakovićPetrov y su extensa bibliografía da cuenta de cada una de ellas. De la lectura de la introducción al libro "Selected Works on Sephardic and Other Jewish Topics" de Kalmi Baruh (2007), escrita por Vidaković-Petrov, se puede concluir que Baruh es una representación de un momento histórico fundamental, donde el hispanista no solo es ese gran intelectual de la comunidad bosnio-judía en Sarajevo, sino el enlace entre España y la región después de más de 400 años de incomunicación política, económica y cultural.

\section{Conclusiones}

El presente artículo busca profundizar en la trayectoria personal y profesional de la investigadora Krinka Vidaković-Petrov a partir de varios encuentros y el acceso a un documento biográfico redactado por ella misma. Por tanto, hago referencia a una persona concreta y a su biografía, a través de una noción de la investigación que es idiográfica; a partir de un fenómeno único o unitario que no puede ser comparado con fenómenos similares. Siendo consciente de las limitaciones metodológicas de este tipo de estudio, me parece relevante emprender un análisis cognitivo del impulso vital que se esconde tras su carrera profesional. Esto invalida cualquier voluntad de buscar describir una etapa histórica determinada, pero solo enfocarse hacia la trayectoria de VidakovićPetrović en la tarea de realizar sus investigaciones sobre Kalmi Baruh y en relación al mundo que la tocó vivir.

Si el contexto familiar encaminó su mundo profesional hacia el hispanismo, las condiciones fueron adversas, tanto desde el punto de vista académico como sociopersonal. En este proceso se cruzaron personas que dirigieron y orientaron sus pasos académicos, incluso viniendo desde diferentes disciplinas académicas, entre la antigua Yugoslavia y España. Se puede concluir, en definitiva, que logró tejer toda una red de contactos que sirvió para confrontar, reconfigurar o sortear los escenarios desfavorables de tal manera que pudiera cumplir con el compromiso intelectual adquirido con los estudios sefardíes. 
Bien es cierto que Yugoslavia se encontraba en una época de aperturismo intelectual y no alineamiento internacional que facilitaba que se tendieran puentes con todo tipo de realidades políticas, culturales y sociales a nivel global. Sin embargo, el análisis de su trayectoria permite detectar su condición de mujer adelantada a su época, avanzando en su carrera académica sin el abrigo institucional de la Universidad de Belgrado, incluso aventajando a la fundación del hispanismo dentro de Yugoslavia, pero también haciendo incursión en una temática desatendida por la academia y por la propia comunidad judía en aquel momento. Al mismo tiempo, esta tarea no tenía apenas recompensas sociales, toda vez que la comunidad judía era muy reducida, y porque indirectamente sus estudios se enfrentaban con la política nacional pro-árabe de la época en Yugoslavia o la enlazaban con España, que en aquella época no tenía relaciones con el régimen de Tito, siendo ambos rivales ideológicos durante la Guerra Fría; situación que no cambiaría hasta bien entrados los años 80 , cuando Vidaković-Petrov publicó su tesis en la editorial Svjetlost (Kultura španskih Jevreja na jugoslovenskom tlu: XVI-XX vek, 1986).

El análisis del perfil autobiográfico y académico de Vidaković-Petrov permite esclarecer varios de los fundamentos que ayudan a comprender la naturaleza aislada $y$ voluntariosa de su trabajo. De las conversaciones mantenidas y del documento al que he tenido acceso, se deducen fuertes convicciones políticas que la conducen hacia el reconocimiento y restablecimiento de injusticias históricas, que en su caso se traducen tanto en su identificación personal como serbia con el drama de la sociedad serbia durante el siglo XX y, por extensión, con el destino dramático de los judíos sefardís de los Balcanes, como con la figura de Kalmi Baruh, uno de sus representantes más insignes, que, además, como ella, era un estudioso del hispanismo. Ella misma declara: “En esos años, investigando el tema sefardí, me sentía como alumna imaginaria de Kalmi Baruh. Hasta recientemente, fui la única que le consideraba padre de los estudios hispánicos modernos en Yugoslavia" (Vidaković-Petrov 2018).

Todo este cuadro mental conduce a una imbricación política de otra naturaleza que encaja en el marco de las aspiraciones personales de juventud de Vidaković-Petrov: la pasión personal por un campo de estudio, el hispanismo, que se personificaba en Kalmi Baruh. En realidad, Baruh es una proyección de sus motivaciones personales y políticas, y una continuación coherente con la vida política y académica que quiso llevar VidakovićPetrov que, como se produjo entre España y los sefardíes balcánicos en la primera mitad del siglo $\mathrm{XX}$, buscaba restablecer relaciones desde que los judíos fueran expulsados de las Coronas de Castilla y León en 1492. El recuerdo de Baruh, tan fáctico como idealizado, otorga a Vidaković-Petrov una vía a través de la que puede plasmar su compromiso con una causa más fuerte que las dificultades que tuvo que afrontar en la vida académica. Para concluir, dice así:

Baruh era mi estrella guiadora personal, el único profesional que me extendía su mano segura, que entendía mi determinación, que podía confirmar que no me 
equivocaba en mi juicio ni en mi amor. Así aparecía Baruh en mi imaginación. ¿Por qué importaba eso? Porque la imaginación es la que nos empuja adelante a atravesar ríos donde todavía no hay puentes y a construir puentes para que los demás puedan pasar, incluso los que carecen del mínimo de imaginación (aquí entra Andrić con su metáfora fenomenal de puentes). (Vidaković-Petrov 2018).

\section{BIBLIOGRAFÍA}

Allport, Gordon W. The Use of Personal Documents in Psychological Science. Prepared for the Committee on Appraisal of Research. New York: Social Science Research Council, 1942. Print.

Capriati, Alejandro. "Tensiones y desafíos en el uso del método biográfico". Cinta de moebio, 60 (2017): 316-327. Revistas académicas de la Universidad de Chile. Web. 6 Ene. 2019.

Denzin, Norman K. Interpretive Biography: Qualitative Research Methods. Book 17. Newbury Park, California: Sage Publications, 1989. Print.

Fox, H.J. "Perspectives on the continuity perspective". International Journal of Aging and Human Development, 14.2 (1981): 97-115. Print.

Pujadas Muñoz, Juan José. El método biográfico: el uso de las historias de vida en las ciencias sociales. Madrid: CIS, 2002. Impreso.

Sanz Hernández, Alexia. "El método biográfico en investigación social: potencialidades y limitaciones de las fuentes orales y los documentos personales". Asclepio, 57.2 (2005): 99-115. Web. 27 Dic. 2018.

Soldatić, Dalibor. "Las literaturas hispánicas en Serbia”. Colindancias, 1 (2010): 21-28. Web. 10 Ene. 2019.

Vidaković-Petrov, Krinka. "Las adivinanzas sefardies de Macedonia". Balkania, 6 (2015): 36-53. Web. 27 Dic. 2018.

- "Kalmi Baruh y los estudios hispánicos modernos en Yugoslavia". Anđelka Pejović et al. (eds.), Estudios hispánicos en la cultura y ciencia Serbia: actas de la Primera conferencia nacional de hispanistas serbios (Facultad de Filología y Artes de Kragujevac, 28-29 noviembre 2014), Kragujevac: Filološko-umetnički fakultet, 2016: 161-169. Impreso.

-. "Una historia que empieza como aventura y termina en nada nuevo". Documento Word inédito. 18 de marzo de 2018.

-. Kultura španskih Jevreja na jugoslovenskom tlu: XVI-XX vek, Svjetlost 1986. Štampano.

- Kalmi Baruh Selected Works on Sephardic and Other Jewish Topics. Jerusalem: Moshe David Gaon Center for Ladino Culture, Shefer Publishers, 2007. Print.

-. "Jevrejske teme u radovima Vojislava Maksimovića". Univerzitet u Istočnom Sarajevu. Filološki Fakultet Pale. Radovi Filoloskog Fakulteta. Broj 12, Knjiga 1. Pal, 2010: 177-192. Štampano. 
Vidaković-Petrov, Krinka. "Kalmi Baruh o Sefardima». Izraz, 5 (1976a): 801-811. Štampano.

-. "Mogućnosti proučavanja jezičko-književnog nasleđa španskih Jevreja na našem tlu" Forum, 7-8 (1976b): 302-312. Štampano.

—. "Jevrejsko-španski listovi u Bosni i Hercegovini" Mostovi, 1977.

-. "Sefardska književnost u Bosni i Hercegovini u svetlu dosadašnjih istraživanja”. Akademija nauka i umjetnosti $\mathrm{BiH}$. Naučni skup Književnost $\mathrm{BiH}$ u svijetlu dosadašnjih istraživanja, Knj. 5, Sarajevo: Posebna izdanja XXXV, 1978: 85-92. Štampano. 